\title{
Article \\ On shape parameter $\alpha$ based approximation properties and $q$-statistical convergence of Baskakov-Gamma operators
}

\author{
Ming-Yu Chen ${ }^{1}\left(\mathbb{D}\right.$, Md. Nasiruzzaman' ${ }^{(D,}$, Mohammad Ayman Mursaleen ${ }^{3,4, *(D)}$ and Nadeem \\ $\operatorname{Rao}^{5}$ iD \\ 1 Fujian Provincial Key Laboratory of Data-Intensive Computing, Key Laboratory of Intelligent Computing \\ and Information Processing, School of Mathematics and Computer Science, Quanzhou Normal University, \\ Quanzhou 362000, China; \\ 2 Department of Mathematics, Faculty of Science, University of Tabuk, PO Box 4279, Tabuk 71491, Saudi \\ Arabia; \\ 3 School of Information and Physical Sciences, The University of Newcastle, Callaghan, New South Wales \\ 2308, Australia; \\ 4 Department of Mathematics and Statistics, Faculty of Science, Universiti Putra Malaysia, 43400 Serdang, \\ Selangor, Malaysia; \\ 5 Department of Mathematics, Shree Guru Gobind Singh Tricentenary University, Gurugram 122505, India.
}

Emails: 2754908504@qq.com; nasir3489@gmail.com; mohdaymanm@gmail.com; nadeemrao1990@gmail.com

* Correspondence: mohdaymanm@gmail.com; Tel.: +39-3348219221

\begin{abstract}
We construct a novel family of summation-integral type hybrid operators in terms of shape parameter $\alpha \in[0,1]$ in this paper. Basic estimates, rate of convergence, and order of approximation are also studied using the Korovkin theorem and the modulus of smoothness. We investigate the local approximation findings for these sequences of positive linear operators utilising Peetre's K-functional, Lipschitz class, and second-order modulus of smoothness.
\end{abstract}

Keywords: Baskakov operators; gamma operators; rate of convergence; Lipschitz maximal space; $q$-density; $q$-statistical convergence.

\section{Introduction}

The theory of linear positive operators deals with question that arise in the approximate representation of an arbitrary function by the simplest one. In the recent past the operator theory is a growing and fascinating field of research in approximation theory with the advent of computer. Several researchers constructed new sequences of linear positive operators to investigate the rapidity of convergence and order of approximation in different function spaces in terms of several generating functions. Recently, Chen et al. [11] constructed a sequence of new linear positive operators known as the $\alpha$-Berstein operators of order $m$ :

$$
T_{m, \alpha}(g ; y)=\sum_{i=0}^{m} g\left(\frac{i}{m}\right) p_{m, i}^{\alpha}(y) \quad(y \in[0,1])
$$

for $g \in C[0,1], m \in \mathbb{N}$ and $\alpha \in[-1,1]$, where $p_{1,0}^{(\alpha)}=1-y, \quad p_{1,1}^{(\alpha)}=y$ and $p_{m, i}^{\alpha}(y)$

$$
=\left[(1-\alpha) y\left(\begin{array}{c}
m-2 \\
i
\end{array}\right)+(1-\alpha)(1-y)\left(\begin{array}{c}
m-2 \\
i-2
\end{array}\right)+\alpha y(1-y)\left(\begin{array}{c}
m \\
i
\end{array}\right)\right] y^{i-1}(1-y)^{m-i-1},
$$

$m \geq 2$.

Remark 1. One can note that for $\alpha=1$, the relation (1) is reduced to classical Bernstein operators [8]. 
These operators are restricted for the space of continuous functions only. To approximate the wider class than the class of continuous function, i.e., space of Lebesgue integrable functions, Mohiuddine et al. constructed Kantorovich-type of $\alpha$-Bernstein operators [19] and Stancu-type $\alpha$-Bernstein-Kantorovich operators [18]. Cai et al. [10] introduced a generalization of classical Bernstein operators based on shape parameter $\alpha \in[0,1]$. These operators are termed as $\alpha$-Bernstein operators of degree $m$ and defined as:

$$
T_{m, \alpha}(g ; y)=\sum_{i=0}^{m} g\left(\frac{i}{m}\right) p_{m, i}^{\alpha}(y), \quad(y \in[0,1])
$$

where $p_{m, i}^{(\alpha)}(y)$ is defined by (2).

Remark 2. Note that, $p_{m, i}^{\alpha}$ in the relation (3) is called $\alpha$-Berstein polynomials of order $m$ and the binomial coefficients

$$
\left(\begin{array}{l}
p \\
q
\end{array}\right)= \begin{cases}\frac{p !}{q !(p-q) !}, & 0 \leq q \leq p \\
0 & \text { otherwise. }\end{cases}
$$

Later on, Aral and Erbay [6] introduced the parametric form of Baskakov-Durrmeyer operators as:

$$
L_{m, \alpha}(g ; w)=\sum_{s=0}^{\infty} P_{m, s}^{\alpha}(w) g\left(\frac{s}{m}\right)
$$

where $f \in C_{B}[0, \infty):=\left\{g \in C[0, \infty):\|g\|=\sup _{0 \leq x<\infty}|g(x)|<\infty\right\}, m \geq 1, w \in[0, \infty)$ and for $\alpha \in[0,1]$

$$
\begin{aligned}
P_{m, s}^{\alpha} g(w) & =\frac{w^{s-1}}{(1+w)^{m+s-1}}\left\{\frac{\alpha w}{1+w}\left(\begin{array}{c}
m+s-1 \\
s
\end{array}\right)-(1-\alpha)(1+w)\left(\begin{array}{c}
m+s-3 \\
s-2
\end{array}\right)\right. \\
& \left.+(1-\alpha) w\left(\begin{array}{c}
m+s-1 \\
s
\end{array}\right)\right\}
\end{aligned}
$$

with $\left(\begin{array}{c}m-3 \\ -2\end{array}\right)=\left(\begin{array}{c}m-2 \\ -1\end{array}\right)=0$. The sequences (4) are restricted for the space of continuous functions only. Motivated by the above development, we construct a sequence of hybrid operators to approximate in a wider class, i.e., the space of Lebesgue integrable functions, as follows:

$$
A_{m, \alpha}^{*}(g ; w)=\sum_{s=0}^{\infty} P_{m, s}^{\alpha}(w) \frac{m^{s+\lambda+1}}{\Gamma(s+\lambda+1)} \int_{0}^{\infty} t^{s+\lambda} e^{-m t} g(t) d t
$$

where $P_{m, S}^{(\alpha)}(w)$ is given by (4) and the gamma function as:

$$
\Gamma(w)=\int_{0}^{\infty} w^{m-1} e^{-w} d w, \quad \Gamma(w)=(w-1) \Gamma(w-1)=(w-1) ! .
$$

In the subsequent sections, we establish basic lemmas, rate of convergence, order of approximation locally and globally in terms of modulus of smoothness, Peetre's K-functional, second modulus of smoothness, Lipschitz space maximal function and weighted modulus of smoothness. Lastly, we study the $q$-statistical convergence. For more basic concepts and related articles we refer to see the published article $[3,5,9,17,21-24,26]$

Remark 3. One can note that, for $\alpha=\lambda=0$, the operators constructed by us in (5) reduced to the classical Baskakov-Durrmeyer operators [7]. 


\section{Basic Estimates and approximation}

Lemma 1. [6] For $m \in \mathbb{N}$, the $\alpha$-Baskakov operator has the following identities:

$$
\begin{aligned}
L_{m, \alpha}(1 ; w) & =1 \\
L_{m, \alpha}(t ; w) & =w+\frac{2}{m}(\alpha-1) w \\
L_{m, \alpha}\left(t^{2} ; w\right) & =w^{2}+\frac{4 \alpha-3}{m} w^{2}+\frac{w}{m^{2}}(m+4 \alpha-4) .
\end{aligned}
$$

Lemma 2. For test function $e_{i}(w)=w^{i}, i \in\{0,1,2\}$, we have the following identities:

$$
\begin{aligned}
A_{m, \alpha}^{*}\left(e_{0} ; w\right) & =1 \\
A_{m, \alpha}^{*}\left(e_{1} ; w\right) & =w+\frac{2}{m}(\alpha-1) w+\frac{\lambda+1}{m} \\
A_{m, \alpha}^{*}\left(e_{2} ; w\right) & =w^{2}\left(1+\frac{4 \alpha-3}{m}\right)+z\left(\frac{2 \lambda+3}{m}+\frac{4 \alpha-4+(2 \lambda+3)(\alpha-1)}{m^{2}}\right) \\
& +\frac{\lambda^{2}+3 \lambda+2}{m^{2}}
\end{aligned}
$$

where $m \in \mathbb{M}$ and $\alpha \in[-1,1]$.

Proof. In view of Lemma 2 , for $e_{0}=1$, we have

$$
\begin{aligned}
A_{m, \alpha}^{*}\left(e_{0} ; z\right) & =\sum_{s=0}^{\infty} P_{m, s}^{\alpha}(w) \frac{m^{s+\lambda+1}}{\Gamma(s+\lambda+1)} \int_{0}^{\infty} t^{s+\lambda} e^{-m t} d t \\
& =\sum_{s=0}^{\infty} P_{m, s}^{\alpha}(w) \frac{m^{s+\lambda+1}}{\Gamma(s+\lambda+1)} \frac{\Gamma(s+\lambda+1)}{m^{s+\lambda+1}} \\
& =1 .
\end{aligned}
$$

For $e_{1}=t$, we have

$$
\begin{aligned}
A_{m, \alpha}^{*}\left(e_{1} ; w\right) & =\sum_{s=0}^{\infty} P_{m, s}^{\alpha}(w) \frac{m^{s+\lambda+1}}{\Gamma(s+\lambda+1)} \int_{0}^{\infty} t^{s+\lambda} e^{-m t} t d t \\
& =\sum_{s=0}^{\infty} P_{m, s}^{\alpha}(w) \frac{m^{s+\lambda+1}}{\Gamma(s+\lambda+1)} \int_{0}^{\infty} t^{s+\lambda+1} e^{-m t} d t \\
& =\sum_{s=0}^{\infty} P_{m, s}^{\alpha}(w) \frac{m^{s+\lambda+1}}{\Gamma(s+\lambda+1)} \frac{\Gamma(s+\lambda+2)}{m^{s+\lambda+2}} \\
& =\sum_{s=0}^{\infty} P_{m, s}^{\alpha}(w) \frac{1}{m} \frac{(s+\lambda+1)}{\Gamma(s+\lambda+1)} \Gamma(s+\lambda+1) \\
& =\sum_{s=0}^{\infty} P_{m, s}^{\alpha}(w) \frac{s}{m}+\frac{\lambda+1}{m} \\
& =z+\frac{2}{m}(\alpha-1) w+\frac{\lambda+1}{m} .
\end{aligned}
$$


For $e_{2}=t^{2}$

$$
\begin{aligned}
& A_{m, \alpha}^{*}\left(e_{2} ; w\right)=\sum_{s=0}^{\infty} P_{m, s}^{\alpha}(w) \frac{m^{s+\lambda+1}}{\Gamma(s+\lambda+1)} \int_{0}^{\infty} t^{s+\lambda} e^{-m t} t^{2} d t \\
& =\sum_{s=0}^{\infty} P_{m, s}^{\alpha}(w) \frac{m^{s+\lambda+1}}{\Gamma(s+\lambda+1)} \int_{0}^{\infty} t^{s+\lambda+2} e^{-m t} d t \\
& =\sum_{s=0}^{\infty} P_{m, s}^{\alpha}(w) \frac{m^{s+\lambda+1}}{\Gamma(s+\lambda+1)} \frac{\Gamma(s+\lambda+3)}{m^{s+\lambda+3}} \\
& =\sum_{s=0}^{\infty} P_{m, s}^{\alpha}(w) \frac{m^{s+\lambda+1}}{\Gamma(s+\lambda+1)} \frac{(s+\lambda+2)(s+\lambda+1)(\Gamma s+\lambda+1)}{m^{s+\lambda+3}} \\
& =\sum_{s=0}^{\infty} P_{m, s}^{\alpha}(w) \frac{(s+\lambda+2)(s+\lambda+3)}{m^{2}} \\
& =\sum_{s=0}^{\infty} P_{m, s}^{\alpha}(w) \frac{s^{2}+(2 \lambda+3) s+\left(\lambda^{2}+3 \lambda+2\right)}{m^{2}} \\
& =\sum_{s=0}^{\infty} P_{m, s}^{\alpha}(w) \frac{s^{2}}{m^{2}}+\frac{(2 \lambda+3) s}{m^{2}}+\frac{\lambda^{2}+3 \lambda+2}{m^{2}} \\
& A_{m, \alpha}^{*}\left(e_{2} ; w\right)=w^{2}+\frac{(4 \alpha-3) w^{2}}{m}+\frac{z(m+4 \alpha-4)}{m^{2}}+\frac{2 \lambda+3}{m}\left(w+\frac{2(\alpha-1) w}{m}\right) \\
& +\frac{\lambda^{2}+3 \lambda+2}{m^{2}} \\
& =w^{2}\left(1+\frac{4 \alpha-3}{m}\right)+w\left(\frac{2 \lambda+3}{m}+\frac{4 \alpha-4+(2 \lambda+3)(\alpha-1)}{m^{2}}\right) \\
& +\frac{\lambda^{2}+3 \lambda+2}{m^{2}}
\end{aligned}
$$

Lemma 3. Let $\eta_{j}(w)=(t-w)^{j}, j \in\{0,1,2\}$. Then,

$$
\begin{aligned}
& A_{m, \alpha}^{*}\left(\eta_{0} ; w\right)=1 \\
& A_{m, \alpha}^{*}\left(\eta_{1} ; w\right)=\frac{2(\lambda-1) w}{m}+\frac{\lambda+1}{m} \\
& A_{m, \alpha}^{*}\left(\eta_{2} ; w\right)=0\left(\frac{1}{m}\right)\left(w^{2}+w+1\right) .
\end{aligned}
$$

Proof. In the light of linearity property of $A_{m, \alpha}^{*}(. ;$.$) and Lemma (2), we get the desired Lemma (3).$

Definition 1. The modulus of continuity for a uniformly continuous function $f$ on $[0, \infty)$ is defined as

$$
\omega(f ; \delta)=\sup _{\left|t_{1}-t_{2}\right| \leq \delta}\left|f\left(t_{1}\right)-f\left(t_{2}\right)\right|, \delta>0, t_{1}, t_{2} \in[0, \infty)
$$

Also, we get

$$
\left|f\left(t_{1}\right)-f\left(t_{2}\right)\right| \leq\left(1+\frac{\left(t_{1}-t_{2}\right)^{2}}{\delta^{2}}\right) \omega(f ; \delta)
$$

Theorem 1. Suppose that $Q_{\varphi}=\left\{\varphi: w \geq 0, \frac{\varphi(w)}{1+w^{2}}\right.$ converges when $\left.w \rightarrow \infty\right\}$. Then, for any $\varphi \in$ $C[0, \infty) \cap Q_{\varphi}$, the operators $A_{m, \alpha}^{*}(. ;$.$) given by (5) converges to function \varphi$ uniformly. 
Proof. Taking into account the property (vi) of Theorem 4.1.4 [4], it is enough to show that

$$
A_{m, \alpha}^{*}\left(e_{j} ; w\right) \rightarrow e_{j}(w), \text { if } j=0,1,2 .
$$

From Lemma 2, we get $A_{m, \alpha}^{*}\left(e_{j} ; w\right) \rightarrow e_{j}(w)$ for $j=0,1,2$ when $m \rightarrow \infty$. Which gives the prove of Theorem 1.

Theorem 2. (See [27]) Let $\mathcal{L}: C[a, b] \rightarrow B[a, b]$ be a linear and positive operator and $f \in C_{B}[a, b]$. Then for $\delta>0$

$$
\begin{aligned}
|(\mathcal{L} f)(x)-f(x)| \leq \quad & f(x)||\left(\mathcal{L} e_{0}\right)(x)-1 \mid \\
& +\left\{\left(\mathcal{L} e_{0}\right)(x)+\delta^{-1} \sqrt{\left(\mathcal{L} e_{0}\right)(x)\left(\mathcal{L} \varphi_{x}^{2}\right)(x)}\right\} \omega_{f}(\delta)
\end{aligned}
$$

where

$$
\varphi_{x}(t)=|t-x|,(x, t) \in[a, b] \times[a, b]
$$

Theorem 3. For any $g \in C_{B}[0, \infty)$, the sequence of operators $A_{m, \alpha}^{*}(. ;$.$) defined by (5) verify the inequality$

$$
\left|A_{m, \alpha}^{*}(g ; w)-g(w)\right| \leq 2 \omega(g ; \delta)
$$

where $\delta=\sqrt{A_{m, \alpha}^{*}\left(\eta_{2} ; w\right)}$ and $C_{B}[0, \infty)$ stands for space of all continuous and bounded functions on the interval $[0, \infty)$.

Proof. In the light of Lemma 2, Lemma 3 and Theorem 2, it is easy to obtain

$$
\left|A_{m, \alpha}^{*}(g ; w)-g(w)\right| \leq\left\{1+\delta^{-1} \sqrt{A_{m, \alpha}^{*}\left(\eta_{2} ; w\right)}\right\} \omega^{*}(g ; \delta)
$$

On taking $\delta=\sqrt{A_{m, \alpha}^{*}\left(\eta_{2} ; w\right)}$, we arrive at the required result.

\section{Local and Global Approximation Results}

The Peetre's K-functional is defined by

$$
K_{2}(g, \delta)=\inf \left\{\|f-h\|+\delta\left\|h^{\prime \prime}\right\|: h \in C_{B}^{2}[0, \infty)\right\},
$$

for any $f \in C_{B}[0, \infty)$ and $\delta>0$, where $C_{B}^{2}[0, \infty)=\left\{h \in C_{B}[0, \infty): h^{\prime}, h^{\prime \prime} \in C_{B}[0, \infty)\right\}$. From DeVore and Lorentz [[13], p.177, Theorem 2.4], there exists an absolute constant $C>0$ such that

$$
K_{2}(f ; \delta) \leq C \omega_{2}(f ; \sqrt{\delta}) .
$$

To prove Theorem 5 , we define the auxiliary operator $\widehat{A}_{m, \alpha}(g ; w)$ as:

$$
\widehat{A}_{(m, \alpha)}(g ; w)=A_{m, \alpha}^{*}(g ; w)+g(w)-g\left(w+\frac{2}{m}(\alpha-1) w+\frac{\lambda+1}{m}\right) .
$$

Theorem 4. For $g \in C_{B}^{2}[0, \infty), w \geq 0$ and $i, \lambda \geq 0, \quad \alpha \in[0,1]$, one gets

$$
\left|\widehat{A}_{m, \alpha}(g ; w)-g(w)\right| \leq \xi_{m}^{w}\left\|g^{\prime \prime}\right\|
$$


where

$$
\xi_{m}^{w}=\widehat{A}_{m, \alpha}\left((t-w)^{2} ; w ; w\right)+\left(\frac{2}{m}(\alpha-1) w+\frac{\lambda+1}{m}\right) .
$$

Proof. From the auxiliary operator (6), we get that

$$
\widehat{A}_{m, \alpha}\left(e_{0} ; w\right)=1, \widehat{A}_{m, \alpha}\left(\eta_{1}(w) ; w\right)=0 \text { and }\left|\widehat{A}_{m, \alpha}(g ; w)\right| \leq 3\|g\| .
$$

From Taylor series expansion, for any $g \in C_{B}^{2}[0, \infty)$, we have

$$
g(t)=g(w)+(t-w) g^{\prime}(w)+\int_{z}^{t}(t-\rho) g^{\prime \prime}(\rho) d \rho .
$$

On operating $\widehat{A}_{m, \alpha}(f ; w)$ in (8), we obtain

$$
\widehat{A}_{m, \alpha}(g ; w)-g(w)=g^{\prime}(w) \widehat{A}_{m, \alpha}(t-w ; w)+\widehat{A}_{m, \alpha}\left(\int_{w}^{t}(t-\rho) g^{\prime \prime}(\rho) d \rho ; w\right) .
$$

Therefore in the view of (7) we get,

$$
\begin{aligned}
\widehat{A}_{m, \alpha}(g ; w)-g(w) & =\widehat{A}_{m, \alpha}\left(\int_{w}^{t}(t-\rho) g^{\prime \prime}(\rho) d \rho ; w\right) \\
& =A_{m, \alpha}^{*}\left(\int_{w}^{t}(t-\rho) g^{\prime \prime}(\rho) d \rho ; z\right) \\
& -\int_{w+\frac{2}{m}(\alpha-1) w+\frac{\lambda+1}{m}}\left(z+\frac{2}{m}(\alpha-1) w+\frac{\lambda+1}{m}-\rho\right) g^{\prime \prime}(\rho) d \rho . \\
\left|\widehat{A}_{m, \alpha}(g ; w)-g(w)\right| & \leq\left|A_{m, \alpha}^{*}\left(\int_{w}^{t}(t-\rho) g^{\prime \prime}(\rho) d \rho ; w\right)\right| \\
& +\left|\int_{w}^{w+\frac{2}{m}(\alpha-1) w+\frac{\lambda+1}{m}}\left(w+\frac{2}{m}(\alpha-1) w+\frac{\lambda+1}{m}-\rho\right) g^{\prime \prime}(\rho) d \rho\right| .
\end{aligned}
$$

Since

$$
\left|\int_{w}^{t}(t-\rho) g^{\prime \prime}(\rho) d \rho\right| \leq(t-w)^{2}\left\|g^{\prime \prime}\right\|
$$

Then

$$
\begin{aligned}
\int_{w}^{w+\frac{2}{m}(\alpha-1) w+\frac{\lambda+1}{m}}\left(w+\frac{2}{m}(\alpha-1) w+\frac{\lambda+1}{m}\right. & -\rho) g^{\prime \prime}(\rho) d \rho \mid \\
& \leq\left(\frac{2}{m}(\alpha-1) w+\frac{\lambda+1}{m}\right)^{2}\left\|g^{\prime \prime}\right\|
\end{aligned}
$$


Combining the equalities (9), (10) and (11), we see that

$$
\begin{aligned}
\left|\widehat{A}_{m, \alpha}(g ; w)-g(w)\right| & \leq\left\{\widehat{A}_{m, \alpha}\left((t-w)^{2} ; w ; w\right)+\left(\frac{2}{m}(\alpha-1) w+\frac{\lambda+1}{m}\right)^{2}\right\}\left\|g^{\prime \prime}\right\| \\
& =\xi_{m}^{w}\left\|g^{\prime \prime}\right\|
\end{aligned}
$$

which completes the proof.

Theorem 5. For any $g \in C_{B}^{2}[0, \infty)$, there exists a positive number $C$ satisfying the inequality

$$
\left|A_{m, \alpha}^{*}(g ; w)-g(w)\right| \leq C \omega_{2}^{*}\left(g ; \sqrt{\xi_{m}^{z}}\right)+\omega\left(g ; \widehat{A}_{m, \alpha}\left(\eta_{1} ; w\right)\right),
$$

where $\xi_{m}^{z}$ is given by Theorem 4.

Proof. Let $h \in C_{B}^{2}[0, \infty)$ and $g \in C_{B}[0, \infty)$. Then using the definition of $\widehat{A}_{m, \alpha}(. ;$.$) , we get$

$$
\begin{aligned}
\left|A_{m, \alpha}^{*}(g ; w)-g(w)\right| & \leq\left|\widehat{A}_{m, \alpha}(g-h ; w)\right|+|(g-h)(w)|+\left|\widehat{D}_{m}^{h}(w)-h(w)\right| \\
& +\left|g\left(w+\frac{2}{m}(\alpha-1) w+\frac{\lambda+1}{m}\right)-g(w)\right| .
\end{aligned}
$$

In view of Theorem 4 and the relations (7), one has

$$
\begin{aligned}
\left|A_{m, \alpha}^{*}(g ; w)-g(w)\right| & \leq 4\|g-h\|+\left|\widehat{A}_{m, \alpha}^{*}(h ; w)-h(w)\right| \\
& +\left|g\left(z+\frac{2}{m}(\alpha-1) w+\frac{\lambda+1}{m}\right)-g(w)\right| \\
& \leq 4\|g-h\|+\xi_{m}(w)\left\|h^{\prime \prime}\right\|+\omega\left(g ; A_{m, \alpha}^{*}\left(\eta_{1} ; w\right)\right) .
\end{aligned}
$$

Using the definition of $K$-functional, we obtain

$$
\left|A_{m, \alpha}^{*}(g ; w)-g(w)\right| \leq C \omega_{2}^{*}\left(g ; \sqrt{\xi_{m}(w)}\right)+\omega^{*}\left(g ; A_{m, \alpha}^{*}\left(\eta_{1} ; z\right)\right) .
$$

This gives the proof of Theorem 5 .

For any fixed two real positive numbers $s_{1}$ and $s_{2}$, the Lipschitz-class of functions [25] is defined by:

$$
\operatorname{Lip}_{M}^{s_{1}, s_{2}}(\beta):=\left\{g \in C_{B}[0, \infty):|g(t)-g(w)| \leq C \frac{|t-w|^{\beta}}{\left(t+s_{1} w+s_{2} w^{2}\right)^{\frac{\beta}{2}}}: w, t \in(0, \infty)\right\}
$$

with the positive constant $C$ and $0<\beta \leq 1$.

Theorem 6. Let $g \in \operatorname{Lip}_{M}^{s_{1}, s_{2}}(\beta)$. Then it follows that

$$
\left|A_{m, \alpha}^{*}(g ; w)-g(w)\right| \leq C\left(\frac{A_{m, \alpha}^{*}\left(\eta_{2} ; w\right)}{s_{1} z+s_{2} z^{2}}\right)^{\frac{\beta}{2}}
$$


where $z>0$.

Proof. For $\beta=1$, we have

$$
\begin{aligned}
\left|A_{m, \alpha}^{*}(g ; w)-g(w)\right| & \leq A_{m, \alpha}^{*}(|g(t)-g(w)| ; w) \\
& \leq C A_{m, \alpha}^{*}\left(\frac{|t-w|}{\left(t+s_{1} w+s_{2} w^{2}\right)^{\frac{1}{2}}} ; w\right)
\end{aligned}
$$

Since $\frac{1}{t+s_{1} w+s_{2} w^{2}}<\frac{1}{s_{1} w+s_{2} w^{2}}$, for every $w \in(0, \infty)$, we get that

$$
\begin{aligned}
\left|A_{m, \alpha}^{*}(g ; w)-g(w)\right| & \leq \frac{M}{\left(s_{1} w+s_{2} w^{2}\right)^{\frac{1}{2}}}\left(A_{m, \alpha}^{*}\left((t-w)^{2} ; w\right)\right)^{\frac{1}{2}} \\
& \leq C\left(\frac{A_{m, \alpha}^{*}\left(\eta_{2} ; w\right)}{s_{1} z+s_{2} w^{2}}\right)^{\frac{1}{2}} .
\end{aligned}
$$

Thus the Theorem 6 holds good when $\beta=1$. Next, on choosing $0<\beta<1$ and applying Hölder's inequality for $p_{1}=\frac{2}{\beta}$ and $p_{2}=\frac{2}{2-\beta}$, we have

$$
\begin{aligned}
\left|A_{m, \alpha}^{*}(g ; w)-g(w)\right| & \leq\left(A_{m, \alpha}^{*}(|g(t)-g(w)| ; z)^{\frac{2}{\beta}}\right)^{\frac{\beta}{2}} \\
& \leq M\left(A_{m, \alpha}^{*}\left(\frac{|t-z|^{2}}{\left(t+s_{1} z+s_{2} z^{2}\right)} ; z\right)\right)^{\frac{\beta}{2}} .
\end{aligned}
$$

Since $\frac{1}{t+s_{1} z+s_{2} z^{2}}<\frac{1}{s_{1} z+s_{2} z^{2}}$ for every $w \in(0, \infty)$, we get

$$
\left|A_{m, \alpha}^{*}(g ; w)-g(w)\right| \leq C\left(\frac{A_{m, \alpha}^{*}\left(\left(|t-w|^{2} ; w\right)\right.}{s_{1} w+s_{2} w^{2}}\right)^{\frac{\beta}{2}}
$$

Thus, we get the proof of Theorem 6.

To obtain the local type approximation results in $r^{\text {th }}$ order, the Lipschitz-maximal function (see Lenze [16]) is given by

$$
\widetilde{\omega}_{r}(g ; w)=\sup _{t \neq w, t \in(0, \infty)} \frac{|g(t)-g(w)|}{|t-w|^{r}}, w \in[0, \infty) \text { and } 0<r \leq 1
$$

Theorem 7. Let $w \in[0, \infty)$ and $g \in C_{B}[0, \infty)$. Then for any $r \in(0,1]$, we have

$$
\left|A_{m, \alpha}^{*}(g ; w)-g(w)\right| \leq \widetilde{\omega}_{r}(g ; w)\left(A_{m, \alpha}^{*}\left(\left(|t-z|^{2} ; z\right)\right)^{\frac{r}{2}} .\right.
$$

Proof. Since we know that

$$
\left|A_{m, \alpha}^{*}(g ; w)-g(w)\right| \leq A_{m, \alpha}^{*}(|g(t)-g(w)| ; z) .
$$

Therefore, from equality (13), we get

$$
\left|A_{m, \alpha}^{*}(g ; w)-g(w)\right| \leq \widetilde{\omega}_{r}(g ; w) A_{m, \alpha}^{*}\left(|t-z|^{r} ; z\right) .
$$


Using the Holder's inequality with $p_{1}=\frac{2}{r}$ and $p_{2}=\frac{2}{2-r}$, we have

$$
\left|A_{m, \alpha}^{*}(g ; w)-g(w)\right| \leq \widetilde{\omega}_{r}(g ; w)\left(A_{m, \alpha}^{*}\left(|t-z|^{2} ; z\right)\right)^{\frac{r}{2}},
$$

which gives the desired result.

From [15], we recall some notation. For the weight function $1+x^{2}$ and $0 \leq x<\infty$, we denote

$$
\begin{gathered}
B_{1+x^{2}}[0, \infty)=\left\{f:|f(x)| \leq M_{f}\left(1+x^{2}\right), M_{f} \text { is a constant depending on } f\right\} \\
C_{1+x^{2}}[0, \infty)=\left\{f \in C[0, \infty):\|f\|_{1+x^{2}}=\sup _{x \in[0, \infty)} \frac{|f|}{1+x^{2}}<\infty\right\}
\end{gathered}
$$

and

$$
C_{1+x^{2}}^{k}[0, \infty)=\left\{f \in C_{1+x^{2}}[0, \infty): \lim _{x \rightarrow \infty} \frac{f(x)}{1+x^{2}}=k, \text { where } k \text { is a constant }\right\} .
$$

Note that $C_{1+x^{2}}[0, \infty) \subset B_{1+x^{2}}[0, \infty)$. Moreover, the modulus of smoothness for any function $\phi$ on closed interval $[0, \mu], \mu>0$ is defined by:

$$
\omega_{\mu}^{*}(\phi, \delta)=\sup _{|t-w| \leq \delta} \sup _{w, t \in[0, \mu]}|\phi(t)-\phi(w)| .
$$

Theorem 8. Let $\omega_{\mu+1}^{*}(\phi ; \delta)$ be the modulus of smoothness defined on $[0, \mu+1] \subset[0, \infty)$. Then for all $\phi \in C_{1+w^{2}}[0, \infty)$

$$
\left\|A_{m, \alpha}^{*}(\phi ; z)-\phi(w)\right\|_{C[0, \mu]} \leq 6 M_{\phi}\left(1+\mu^{2}\right) \delta_{m}(\mu)+2 \omega_{\mu+1}^{*}\left(\phi ; \sqrt{\delta_{m}(\mu)}\right),
$$

where $\delta_{m}(\mu)=A_{m, \alpha}^{*}\left(\eta_{2} ; \mu\right)$.

Proof. For all $w \in[0, \mu]$ and $t \in[0, \infty)$, one has

$$
|\phi(t)-\phi(w)| \leq 6 M_{\phi}\left(1+\mu^{2}\right)(t-w)^{2}+\left(1+\frac{|t-z|}{\delta}\right) \omega_{\mu+1}^{*}(\phi ; \delta) .
$$

On applying the operators $A_{m, \alpha}^{*}$, we see that

$$
\left|A_{m, \alpha}^{*}(\phi ; w)-\phi(w)\right| \leq 6 M_{\phi}\left(1+\mu^{2}\right) A_{m, \alpha}^{*}\left((t-w)^{2} ; w\right)+\left(1+\frac{A_{m, \alpha}^{*}(|t-w| ; w)}{\delta}\right) \omega_{\mu+1}^{*}(\phi ; \delta) .
$$

Thus, for $w \in[0, \mu]$, applying the Lemma (2), we get

$$
\left|A_{m, \alpha}^{*}(\phi ; w)-\phi(w)\right| \leq 6 M_{\phi}\left(1+\mu^{2}\right) \delta_{m}(\mu)+\left(1+\frac{\sqrt{\delta_{m}(\mu)}}{\delta}\right) \omega_{\mu+1}^{*}(\phi ; \delta) .
$$

Taking $\delta=\delta_{m}(\mu)$, we get the desired result.

Theorem 9. Suppose the operators $A_{m, \alpha}^{*}(. ;$.$) acting from C_{1+w^{2}}^{k}[0, \infty)$ to $B_{1+w^{2}}[0, \infty)$. Then, for $\phi \in$ $C_{1+w^{2}}^{k}[0, \infty)$, we have

$$
\lim _{m \rightarrow \infty}\left\|A_{m, \alpha}^{*}(\phi)-\phi\right\|_{1+w^{2}}=0 .
$$

Proof. For the results of Theorem 9, we have to show that 


$$
\lim _{m \rightarrow \infty}\left\|A_{m, \alpha}^{*}\left(e_{i}\right)-w^{i}\right\|_{1+w^{2}}=0, i=0,1,2
$$

By using the Lemma 2, it is enough to show

$$
\left\|A_{m, \alpha}^{*}\left(e_{0}\right)-w^{0}\right\|_{1+w^{2}}=\sup _{w \in[0, \infty)} \frac{\left|A_{m, \alpha}^{*}\left(e_{0} ; w\right)-1\right|}{1+w^{2}}=0 \text { for } i=0 .
$$

For $i=1$,

$$
\begin{aligned}
\left\|A_{m, \alpha}^{*}\left(e_{1}\right)-w^{1}\right\|_{1+w^{2}} & =\sup _{w \in[0, \infty)} \frac{\frac{2}{m}(\alpha-1) w+\frac{\lambda+1}{m}}{1+w^{2}} \\
& =\frac{2}{m}(\alpha-1) \sup _{w \in[0, \infty)} \frac{w}{1+w^{2}}+\frac{\lambda+1}{m} \sup _{w \in[0, \infty)} \frac{1}{1+w^{2}}
\end{aligned}
$$

which gives us $\left\|A_{m, \alpha}^{*}\left(e_{1}\right)-w^{1}\right\|_{1+w^{2}} \rightarrow 0$ as $m \rightarrow \infty$. For $i=2$,

$$
\begin{aligned}
\left\|A_{m, \alpha}^{*}\left(e_{2}\right)-w^{2}\right\|_{1+w^{2}} & =\sup _{w \in[0, \infty)} \frac{\left|w^{2}\left(\frac{4 \alpha-3}{m}\right)+w\left(\frac{2 \lambda+3}{m}+\frac{4 \alpha-4+(2 \lambda+3)(\alpha-1)}{m^{2}}\right)\right|}{1+w^{2}} \\
& +\sup _{w \in[0, \infty)} \frac{\left|\frac{\lambda^{2}+3 \lambda+2}{m^{2}}\right|}{1+w^{2}} \\
& =\frac{4 \alpha-3}{m} \sup _{w \in[0, \infty)} \frac{w^{2}}{1+z^{2}} \\
& +\left(\frac{2 \lambda+3}{m}+\frac{4 \alpha-4+(2 \lambda+3)(\alpha-1)}{m^{2}}\right) \sup _{w \in[0, \infty)} \frac{w}{1+w^{2}} \\
& +\frac{(\lambda+1)(\lambda+2)}{m^{2}} \sup _{w \in[0, \infty)} \frac{1}{1+w^{2}},
\end{aligned}
$$

and we get $\left\|A_{m, \alpha}^{*}\left(e_{2}\right)-w^{2}\right\|_{1+w^{2}} \rightarrow 0$ as $m \rightarrow \infty$. Hence we get the result.

Here, we study the approximation of locally integrable functions belongs to $C_{1+w^{2}}^{k}[0, \infty)$. Such type of result is investigated by Gadjiev [15].

Theorem 10. Suppose $\phi \in C_{1+w^{2}}^{k}[0, \infty)$. Then for any $\theta>0$, we have

$$
\lim _{m \rightarrow \infty} \sup _{w \in[0, \infty)} \frac{\left|A_{m, \alpha}^{*}(\phi ; w)\right|-\phi(w)}{\left(1+w^{2}\right)^{1+\theta}}=0 .
$$


Proof. Let $w_{0}$ be the fixed positive real number. Then

$$
\begin{aligned}
\sup _{w \in[0, \infty)} \frac{\left|A_{m, \alpha}^{*}(\phi ; w)\right|-\phi(w)}{\left(1+w^{2}\right)^{1+\theta}} & \leq \sup _{w \leq w_{0}} \frac{\left|A_{m, \alpha}^{*}(\phi ; w)\right|-\phi(w)}{\left(1+w^{2}\right)^{1+\theta}}+\sup _{w \geq w_{0}} \frac{\left|A_{m, \alpha}^{*}(\phi ; w)\right|-\phi(w)}{\left(1+z^{2}\right)^{1+\theta}} \\
& \leq\left\|A_{m, \alpha}^{*}(\phi ; w) \mid-\phi(w)\right\|_{C\left[0, z_{0}\right]} \\
& +\|\phi\|_{1+w^{2}} \sup _{w \geq w_{0}} \frac{\left|A_{m, \alpha}^{*}\left(1+t^{2} ; w\right)\right|}{\left(1+w^{2}\right)^{1+\theta}}+\sup _{w \geq w_{0}} \frac{|\phi(w)|}{\left(1+w^{2}\right)^{1+\theta}} \\
& =I_{1}+I_{2}+I_{3}(\text { say }) .
\end{aligned}
$$

Since $|\phi(w)| \leq\|\phi\|_{1+w^{2}}\left(1+w^{2}\right)$, we get

$$
\begin{aligned}
I_{3} & =\sup _{w \geq w_{0}} \frac{|\phi(w)|}{\left(1+w^{2}\right)^{1+\theta}} \\
& \leq \sup _{w \geq w_{0}} \frac{\|\phi\|_{1+w^{2}}\left(1+w^{2}\right)}{\left(1+w^{2}\right)^{1+\theta}} \leq \frac{\|\phi\|_{1+w^{2}}}{\left(1+w^{2}\right)^{\theta}} .
\end{aligned}
$$

For an arbitrary real number $\epsilon>0$, in view of Theorem 1 , there exists $m_{1} \in \mathbb{N}$ satisfying

$$
\begin{aligned}
I_{2} & <\frac{1}{\left(1+w^{2}\right)^{\theta}}\|\phi\|_{1+w^{2}}\left(1+w^{2}+\frac{\epsilon}{3\|\phi\|_{1+w^{2}}}\right) \text { for all } m_{1} \geq m \\
& <\frac{\|\phi\|_{1+w^{2}}}{\left(1+w^{2}\right)^{\theta}}+\frac{\epsilon}{3}, \text { for all } m_{1} \geq m
\end{aligned}
$$

This implies that

$$
I_{2}+I_{3}<2 \frac{\|\phi\|_{1+w^{2}}}{\left(1+w^{2}\right)^{\theta}}+\frac{\epsilon}{3}
$$

For any sufficiently large number $w_{0}$, we have $\frac{\|\phi\|_{1+w^{2}}}{\left(1+w^{2}\right)^{\theta}}<\frac{\epsilon}{6}$.

$$
I_{2}+I_{3}<\frac{2 \epsilon}{3} \text { for all } m_{1} \geq m
$$

In the light of Theorem 9 and for any $m_{2}>m$, one has

$$
I_{1}=\left\|A_{m, \alpha}^{*}(\phi ; w)-\phi\right\|_{C\left[0, z_{0}\right]}<\frac{\epsilon}{3} \text { for all } m_{2} \geq m
$$

Take $m_{3}=\max \left(m_{1}, m_{2}\right)$ and taking (14) by values of $I_{1}, I_{2}, I_{3}$ we easily get

$$
\sup _{w \in[0, \infty)} \frac{\left|A_{m, \alpha}^{*}(\phi ; w)\right|-\phi(w)}{\left(1+w^{2}\right)^{1+\theta}}<\epsilon
$$

Thus, the proof of Theorem 10 is completed.

\section{4. q-Density and q-statistical convergence}

Recently, the $q$-analog of density and statistical convergence are studied in [2]. Let $\mathcal{E} \subseteq \mathbb{N}$ (the set of natural numbers). Then the $q$-density is defined by

$$
\delta_{q}(\mathcal{E})=\delta_{C_{1}^{q}}(\mathcal{E})=\lim \inf _{n \rightarrow \infty}\left(C_{1}^{q} \chi_{\mathcal{E}}\right)_{n}=\lim \inf _{n \rightarrow \infty} \sum_{k \in \mathcal{E}} \frac{q^{k-1}}{[n]}, q \geq 1,
$$


where $\mathcal{C}^{1}(q)=\left(c_{n k}^{1}\left(q^{k}\right)\right)_{n, k=0}^{\infty}$ is the $q$-Cesàro matrix (see [1], [2]) defined by

$$
c_{n k}^{1}\left(q^{k}\right)= \begin{cases}\frac{q^{k}}{[n+1]_{q}} & \text { if } k \leq n \\ 0 & \text { otherwise. }\end{cases}
$$

which is regular for $q \geq 1$, where the $q$-integer $(q>0)$ of any positive integer $n$ is defined by

$$
[n]=[n]_{q}= \begin{cases}\frac{1-q^{n}}{1-q}, & q \neq 1, \\ n, & q=1 .\end{cases}
$$

A sequence $\eta=\left(\eta_{k}\right)$ is said to be $q$-statistically convergent to the number $\mathcal{L}$ if $\delta_{q}\left(\mathcal{L}_{\varepsilon}\right)=0$, where $\mathcal{L}_{\varepsilon}=\left\{k \leq n:\left|\eta_{k}-l\right| \geq \varepsilon\right\}$ for every $\varepsilon>0$. That is,

$$
\lim _{n} \frac{1}{[n]} \#\left\{k \leq n: q^{k-1}\left|\eta_{k}-l\right| \geq \varepsilon\right\}=0
$$

and we write $S t_{q}-\lim \eta_{k}=l$.

If $\delta(\mathcal{E})=0$ for an infinite set $\mathcal{E}$ then $\delta_{q}(\mathcal{E})=0$, hence statistical convergence [14] implies $q$-statistical convergence but not conversely (c.f. [2, Example 15]).

Theorem 11. For $\phi \in C_{1+z^{2+\lambda}}^{k}[0, \infty)$, we have

$$
S t_{q}-\lim _{m}\left\|A_{m, \alpha}^{*}(\phi ; w)-\phi\right\|_{1+w^{2+\lambda}}=0, \text { for all } \lambda>0 .
$$

Proof. For $\lambda=0$, it is easy to get that

$$
S t_{q}-\lim _{m}\left\|A_{m, \alpha}^{*}\left(e_{i} ; w\right)-e_{i}\right\|_{1+w^{2}}=0, \text { for } i \in\{0,1,2\} .
$$

From Lemma 2, we see that

$$
\begin{aligned}
\left\|A_{m, \alpha}^{*}\left(e_{1} ; w\right)-w\right\|_{1+w^{2}} & =\sup _{w \in[0, \infty)} \frac{w}{1+w^{2}}\left|\frac{2}{m}(\alpha-1)\right| \\
& +\sup _{w \in[0, \infty)} \frac{1}{1+w^{2}}\left|\frac{\lambda+1}{m}\right| \\
& =\frac{2}{m}(\alpha-1) \sup _{w \in[0, \infty)} \frac{w}{1+w^{2}} \\
& +\frac{\lambda+1}{m} \sup _{w \in[0, \infty)} \frac{1}{1+w^{2}} .
\end{aligned}
$$

Now, for a given positive $\epsilon>0$, let

$$
\begin{aligned}
& M_{1}:=\left\{m:\left\|A_{m, \alpha}^{*}\left(e_{1} ; w\right)-w\right\| \geq \epsilon\right\}, \\
& M_{2}:=\left\{m: \frac{2}{m}(\alpha-1) \geq \frac{\epsilon}{2}\right\}, \\
& M_{3}:=\left\{m: \frac{\lambda+1}{m} \geq \frac{\epsilon}{2}\right\} .
\end{aligned}
$$


This implies that $M_{1} \subseteq M_{2} \cup M_{3}$ which shows that $\delta_{q}\left(M_{1}\right) \leq \delta_{q}\left(M_{2}\right)+\delta_{q}\left(M_{3}\right)$. Hence, we have

$$
S t_{q}-\lim _{m}\left\|A_{m, \alpha}^{*}\left(e_{1} ; w\right)-w\right\|_{1+w^{2}}=0 .
$$

For $i=2$ in the view of Lemma 2, we get

$$
\begin{aligned}
\left\|A_{m, \alpha}^{*}\left(e_{2} ; w\right)-w^{2}\right\|_{1+w^{2}} & =\sup _{w \in[0, \infty)} \frac{1}{1+w^{2}} \mid w^{2}\left(\frac{4 \alpha-3}{m}\right) \\
& +w\left(\frac{2 \lambda+3}{m}+\frac{4 \alpha-4+(2 \lambda+3)(\alpha-1)}{m^{2}}\right)+\frac{(\lambda+1)(\lambda+2)}{m^{2}} \mid \\
& =\frac{4 \alpha-3}{m} \sup _{w \in[0, \infty)} \frac{w}{1+w^{2}}+\frac{2 \lambda+3}{m} \sup _{w \in[0, \infty)} \frac{w^{2}}{1+w^{2}} \\
& +\frac{4 \alpha-4+(2 \lambda+3)(\alpha-1)}{m^{2}} \sup _{w \in[0, \infty)} \frac{w}{1+w^{2}} \\
& +\frac{(\lambda+1)(\lambda+2)}{m^{2}} \sup _{w \in[0, \infty)} \frac{1}{1+w^{2}} .
\end{aligned}
$$

For a given positive $\epsilon>0$, let

$$
\begin{aligned}
& T_{1}:=\left\{m:\left\|A_{m, \alpha}^{*}\left(e_{2} ; w\right)-w^{2}\right\| \geq \epsilon\right\}, \\
& T_{2}:=\left\{m: \frac{4 \alpha-3}{m} \geq \frac{\epsilon}{4}\right\}, \\
& T_{3}:=\left\{m: \frac{2 \lambda+3}{m} \geq \frac{\epsilon}{4}\right\}, \\
& T_{4}:=\left\{m: \frac{4 \alpha-4+(2 \lambda+3)(\alpha-1)}{m^{2}} \geq \frac{\epsilon}{4}\right\}, \\
& T_{5}:=\left\{m: \frac{(\lambda+1)(\lambda+2)}{m^{2}} \geq \frac{\epsilon}{4}\right\} .
\end{aligned}
$$

Then $T_{1} \subseteq T_{2} \cup T_{3} \cup T_{4} \cup T_{5}$, and we get

$$
\delta_{q}\left(T_{1}\right) \leq \delta_{q}\left(T_{2}\right)+\delta_{q}\left(T_{3}\right)+\delta_{q}\left(T_{4}\right)+\delta_{q}\left(T_{5}\right)
$$

Letting $m \rightarrow \infty$, we have

$$
S t_{q}-\lim _{m}\left\|A_{m, \alpha}^{*}\left(e_{2} ; w\right)-w^{2}\right\|_{1+w^{2}}=0 .
$$

This gives the desired proof of Theorem 11.

\section{Declarations}

\section{Conflicts of Interest}

The authors declare that they have no conflicts of interest. 


\section{Authors' contributions}

All authors contributed equally to writing this paper. All authors read and approved the manuscript.

\section{Data Availability}

No data were used to support this study.

\section{Acnowledgement}

The third author is supported by the JADD program (by UPM and UoN).

\section{Funding}

No funding was provided for this research.

\section{References}

1. Akgun, F.A.; Rhoades, B.E. Properties of some $q$-Hausdorff matrices. Appl. Math. Comput.. 2013, 219, 7392-7397, DOI: https://doi.org/10.1016/j.amc.2013.01.016.

2. Aktuğlu, H.; Bekar, Ş. q-Cesàro matrix and q-statistical convergence. Jour. Comput. Appl. Math.. 2011, 235, 4717-4723, DOI: https://doi.org/10.1016/j.cam.2010.08.018.

3. Alotaibi, A.; Nasiruzzaman, M.; Mursaleen, M. Approximation by Phillips operators via $q$-Dunkl generalization based on a new parameter. Journal of King Saud University-Science. 2021, 33, 101413, DOI: https://doi.org/10.1016/j.jksus.2021.101413.

4. Altamore, F.; Campiti, M. Korovkin-type approximation theory and its applications. Vol 17. Walter De Gruyter, Berlin (Gruyter Studies in Mathematics, Appendix A By Michael Pannenberg and Appendix B By Fendinand Beckho), 1994.

5. Al-Abied, A. A. H. A.; Ayman Mursaleen, M.; Mursaleen, M. Szász type operators involving Charlier polynomials and approximation properties. Filomat. 2021, 35(15), 5149-5159, DOI: https://doi.org/10.2298/FIL2115149A.

6. Aral, A.; Erbay, H. Parametric generalization of Baskakov operators. Math. Commun.. 2019, 24, 119-131.

7. Baskakov, V. An instance of a sequence of linear positive operators in the space of continuous functions. Doklady Akademii Nauk SSSR. 1957, 113, 249-251.

8. Berstein, S.N. Démonstration du théorém de Weierstrass fondée sur le calcul des probabilitiés. Commun Kharkov Math Soc.. 1912/13, 13, 1-2.

9. Cai, Q. B.; Kilicman, A.; Ayman Mursaleen, M. Approximation properties and $q$-statistical convergence of Stancu type generalized Baskakov-Szász operators. Journal of Function Spaces. 2022, 2022, 2286500, DOI: https://doi.org/10.2298/FIL2115149A.

10. Cai, Q. B.; Lian, B. Y.; Zhou G. Approximation Properties of $\lambda$-Bernstein operators. J. Inequal. Appl. 2018. 2018, 2018, 61, DOI: https://doi.org/10.1186/s13660-018-1653-7.

11. Chen, X.; Tan, J.; Liu, Z.; Xie, J. Approximation of functions by a new family of generalized Bernstein operators. J. Math. Anal. Appl.. 2017, 450, 244-261, DOI: https://doi.org/10.1016/j.jmaa.2016.12.075.

12. Connor, J.S. On strong matrix summability with respect to a modulus and statistical convergence. Canad. Math. Bull.. 1989, 32, 194-198, DOI: https:/ /doi.org/10.4153/CMB-1989-029-3.

13. DeVore, R. A.; Lorentz, G. G. Constructive Approximation. Grudlehren der Mathematicshen Wissenchaften, Fundamental principales of mathematical sciences, Springer, Berlin, 1993.

14. Fast, H. Sur la convergence statistique. Colloq. Math.. 1951, 2, 241-244.

15. Gadziev, A. D. Theorem of the type of P. P. Korovkin's theorems. Mat. Zametki. 1976 20(5), 781-786.

16. Lenze, B. On Lipschitz type maximal functions and their smoothness spaces. Nederl Akad Indag Math. 1988, 50, 53-63, DOI: https://doi.org/10.1016/1385-7258(88)90007-8.

17. Kilicman, A.; Mursaleen, M. A.; Al-Abied, A. A. H. A. Stancu type Baskakov-Durrmeyer operators and approximation properties. Mathematics. 2020, 8(7), 1164, DOI: https://doi.org/10.3390/math8071164.

18. Mohiuddine, S. A.; Özge, F. Approximation of functions by Stancu variant of Bernstein-Kantorovich operators based on shape parameter $\alpha$. Rev. R. Acad. Cienc. Exactas Fis. Nat. Ser. A Math. RACSAM. 2020 114, 70, DOI: https://doi.org/10.1007/s13398-020-00802-w. 
19. Mohiuddine, S. A.; Acar, T.; Alotaibi A. Construction of a new family of Bernstein-Kantorovich operators. Math. Meth. Appl. Sci. 2017, 40, 7749-7759, DOI: https://doi.org/10.1002/mma.4559.

20. Mohiuddine, S.A. Statistical weighted $A$-summability with application to Korovkin's type approximation theorem. J. Inequal. Appl.. 2016, 2016, 101, DOI: https://doi.org/10.1186/s13660-016-1040-1.

21. Mursaleen, M.; Ansari, K. J.; Khan, A. Approximation properties and error estimation of $q$-Bernstein shifted operators. Numerical Algorithms. 2020, 84, 207-227, DOI: https://doi.org/10.1007/s11075-019-00752.

22. Nasiruzzaman, M.; Aljohani, A.F. Approximation by Szász-Jakimovski-Leviatan type operators via aid of Appell polynomials. Journal of function spaces. 2020, 2020, 9657489, DOI: https:/ / doi.org/10.1155/2020/9657489.

23. Nasiruzzaman, M.; Aljohani, A.F. Approximation by parametric extension of Szász-Mirakjan-Kantorovich operators involving the Appell polynomials. Journal of Function Spaces. 2020, 2020, 9657489, DOI: https:/ / doi.org/10.1155/2020/8863664.

24. Nasiruzzaman, M. Approximation properties by Szász operators to bivariate functions via Dunkl analogue Iranian Jour. Sci. Tech. (Sci.). 2021, 45, 259-269, DOI: https://doi.org/10.1007/s40995-020-01018-8.

25. Ozarslan, M. A.; Aktuğlu, H. Local approximation for certain King type operators. Filomat. 2013, 27, 173-181, DOI: https://doi.org/10.2298/FIL1301173O.

26. Rao, N.; Wafi, A.; Acu, A.M. q-Szász-Durrmeyer type operators based on Dunkl analogue. Complex Anal. Oper. Theory. 2019, 13(3), 915-934, DOI: https://doi.org/10.1007/s11785-017-0647-7.

27. Shisha, O.; Bond B. The degree of convergence of linear positive operators. Proc. Nat. Acad. Sci. USA. 1968, 60(4), 1196-1200, DOI: https://doi.org/10.1073/pnas.60.4.1196. 